\title{
Uso de naltrexona en la deshabituación de pacientes con dependencia de alcohol en el estado español
}

\author{
Izouierdo, M. '; Rodriguez-Martos, A. ${ }^{2}$; Llopis, J. ${ }^{3}$; Cañuelo, B. ${ }^{4}$; Guigou, G. ${ }^{5}$; Izouierdo, J. ${ }^{6}$; Lorenzo, A.7; \\ MATeO, M. ${ }^{8}$; Rubio, G. ${ }^{9}$; VARO, J. ${ }^{10}$ \\ (1) Unidad de Alcoholismo.Complejo Sanitario Provincial. Plasencia. \\ (2) Plan de Acción sobre Drogas de Barcelona. \\ (3) Unidad de Conductas Adictivas. Centro de Salud San Agustin. Castellón. \\ (4) Centro de Toxicomanias Cruz Roja. Cordoba. \\ (5) CAD Sta. Cruz de Tenerife. \\ (6) Consell Insular de Mallorca. Centro Comarcal Palma Periferia. Palma de Mallorca. \\ (7) Servicio de Psiquiatría. Hospital General de Galicia. Santiago de Compostela. \\ (8) Hospital Divino Vallés. Burgos. \\ (9) Hospital Psiquiátrico Sagrado Corazón de Jesús. Madrid \\ (10) Centro de Salud Mental de Ermitagaña. Pamplona.

\section{RESUMEN}

Objetivos: Describir el perfil del paciente alcohólico que más se beneficiaría del tratamiento con naltrexona y la utilidad del mismo para disminuir el deseo de beber, las recaídas y los episodios de consumo. Métodos: Estudio multicéntrico, observacional, de diseño naturalístico. Se estudiaron 321 pacientes con dependencia de alcohol (DSM-IV), tratados con naltrexona y seguidos durante 9 meses. Resultados: El estudio del perfil de paciente respondedor/no respondedor sólo mostró diferencias respecto a nivel de estudios $(p=0,039)$ y situación laboral $(p=0,041)$. El análisis de resultados reveló los siguientes índices: abstinencia total ó ningún consumo de alcohol en el 45,4\%; algún consumo en el 7,2\%; y recaída en el $27,3 \%$. Discusión: No ha sido posible describir el perfil de paciente alcohólico que más se beneficiaría del uso de naltrexona. Las limitaciones del estudio derivan de la heterogeneidad de la muestra y ausencia de grupo control con asignación aleatoria de casos. Se requieren más estudios capaces de identificar factores predictores de respuesta terapéutica. En cualquier caso, este estudio no sólo ha confirmado la utilidad de la naltrexona para disminuir la cantidad y los días de consumo de alcohol, sino que su largo seguimiento (9 meses) ha permitido comprobar también el beneficio adicional de un tratamiento prolongado. A los 9 meses, aumenta el porcentaje de pacientes que controlan el consumo sin cumplir criterios de recaída.

Palabras clave: dependencia alcohólica, abstinencia, reanudación del consumo, recaída, naltrexona.

\section{ABSTRACT}

Objectives: to describe the profile of alcoholic patients who most benefit from naltrexone treatment, and to show its utility in decreasing craving, relapses and consumption episodes. Methods: multicentre and observational study with a naturalistic design. 321alcohol- dependent patients (DSM-IV) treated with naltrexone were studied and followed up over 9 months. Results: the responder/ non-responder patient profile showed only differences concerning the educational level $(p=0.039)$ and work situation $(p=0.041)$. The outcome analysis showed the following percentages: $45.4 \%$ total abstinence or absence of alcohol consumption; $7.2 \%$ some consumption and $27.3 \%$ relapses. Discussion: It has not been possible to describe the profile of the alcoholic patient who would benefit the most from naltrexone. Limitations of the study derive from the heterogeneous sample and from the lack of a control group with random allocation of cases. More studies are needed in order to identify the predictive factors for a therapeutic response. However, this study has not only confirmed the usefulness of naltrexone in decreasing the quantity and frequency of alcohol consumption, but its long term follow-up (9 months) allowed verification of the additional benefit of a protracted treatment. After 9 months, there is also an increase in the number of patients who are able to control their consumption without fulfilling relapse criteria.

Key words: Alcohol dependence, abstinence, resumption of consumption, relapse, naltrexone.

Las características fundamentales de la dependencia de alcohol son: la preocupación para conseguirlo, su consumo compulsivo a pesar de las consecuencias adversas que conlleva y la recaída o la incapacidad de abstenerse a pesar de sus efectos negativos ${ }^{1}$. 
Son muchos los factores que determinan el inicio y la evolución de los trastornos relacionados con el consumo del alcohol, por lo que su abordaje terapeútico resulta díficil. Las teorías más aceptadas sobre la génesis de la dependencia de alcohol, refieren una interrelación entre los factores genéticos, biológicos, ambientales, psicológicos y sociales ${ }^{2,3}$.

Todas estas premisas justifican la afirmación de que "la dependencia de alcohol se caracteriza por la gran variabilidad de perfiles en los individuos afectados". Las diferencias entre los pacientes son tan importantes que resulta díficil establecer un "perfil" de paciente que se ajuste a un patrón común. De ahí que el modelo de intervención terapéutica se deba ajustar perfectamente a las características individuales de cada paciente, obligando al facultativo a distinguir claramente las distintas fases del tratamiento, y actuar de diferente forma según la fase del mismo ${ }^{4}$.

Es importante señalar que en las últimas décadas nuevas evidencias experimentales y clínicas han ampliado el conocimiento de las bases neurobiológicas que determinan la relación entre determinados sistemas de neurotransmisión cerebral y la regulación del consumo de alcohol ${ }^{5}$, lo que ha permitido el uso terapeútico de nuevos fármacos que mejoran la eficacia de los programas de tratamiento y que aumentan las tasas de abstinencia a largo plazo.

En base a estos descubrimientos han surgido fármacos como los antagonistas de los receptores opiáceos los cuales, en combinación con intervenciones psicosociales, mejoran las tasas de éxito del tratamiento de la dependencia de alcohol. Entre ellos se incluye el principio activo naltrexona, un antagonista competitivo de los receptores opiáceos que se caracteriza por presentar una acción prolongada.

Los estudios preclínicos sugieren que naltrexona es un efectivo agente farmacológico en el tratamiento de la dependencia de alcohol. Los citados estudios preclínicos ponen de manifiesto que mientras que pequeñas dosis de morfina incrementan la consumición voluntaria de alcohol en animales ${ }^{6,7}$, los antagonistas opiáceos como naloxona y naltrexona reducen claramente el consumo de alcohol en diversas circunstancias $^{7-11}$.

Análogamente, diferentes estudios clínicos 12-20,23,25 han evidenciado la utilidad terapéutica de la naltrexona en el abordaje terapéutico de la dependencia de alcohol. Los resultados de estos estudios muestran que, en comparación con placebo, los pacientes tratados con naltrexona presentan una reducción significativa tanto del deseo de beber como del número de recaídas. Por consiguiente se establece que la naltrexona constituye una arma terapéutica adecuada para colaborar en el proceso de recuperación de los pacientes con dependencia de alcohol.
Sin embargo, y a pesar de todas estas evidencias clínicas, se observa una marcada escasez de estudios a largo plazo sobre el uso de naltrexona en la deshabituación de pacientes con dependencia de alcohol. Esta situación motivó el desarrollo a nivel nacional del estudio objeto del presente artículo.

El objetivo principal del presente trabajo ha sido siempre eminentemente práctico, a la vez que ha pretendido recoger el máximo de información acerca del perfil del paciente, lo cual resulta de gran utilidad a la hora de conseguir mejores resultados terapéuticos tanto a corto como a largo plazo. Por ello, y pese a las limitaciones que se han constatado en estudios análogos realizados hasta el momento en este ámbito, constituyeron objetivos secundarios del presente estudio:

1. Comprobar el beneficio de la adición de naltrexona a las técnicas habituales de tratamiento del enfermo con dependencia de alcohol para mejorar la deshabituación;

2. Evaluar la capacidad de la naltrexona de disminuir el deseo de beber ("craving"), consolidar la deshabituación y prevenir las recaídas;

3. Medir la capacidad de la naltrexona para disminuir el número de episodios de reanudación de consumo de alcohol, la frecuencia del consumo, la intensidad del consumo y la duración de los episodios de consumo;

4. Delimitar la duración temporal recomendable del tratamiento de deshabituación con naltrexona;

5. Evaluar la seguridad del uso de naltrexona en un grupo de pacientes con dependencia de alcohol en tratamiento de deshabituación;

6. Comparar la utilidad de la naltrexona asociada a técnicas habituales de intervención frente al empleo de estas técnicas solas en el mantenimiento de la abstinencia de aquellos pacientes del estudio que abandonaran voluntariamente el tratamiento farmacológico.

\section{MATERIAL Y MÉTODOS}

El trabajo de campo se realizó sobre un total de 321 pacientes. Todos ellos cumplían criterios de DSMIV de dependencia de alcohol y presentaban las siguientes características basales: mayoritariamente varones (80\%), edad media 41,74 \pm 9,89 años; con trastorno de dependencia alcohólica moderada $(53,8 \%)$ o grave $(40,1 \%)$ y de dependencia fisiológica ( $72,1 \%$ de los pacientes); media de consumo semanal de alcohol en el último trimestre de 133,62 UBE $( \pm 293,5)$. El $64,2 \%$ de los casos realizaba este consumo de forma regular. La antigüedad de la dependencia alcohólica se estableció en más de 5 años en un 
76,4\% de los casos. Un 54\% de los pacientes había realizado tratamientos previos para la deshabituación alcohólica.

Todos los pacientes incluidos en el estudio recibieron tratamiento con naltrexona, realizándose a continuación un seguimiento de 9 meses de duración, período durante el cual se realizaron 6 visitas de seguimiento (la inicial y 5 para el control de la evolución de los parámetros seleccionados), con independencia de las entrevistas terapéuticas que el investigador consideró oportuno realizar. Las visitas de control se realizaron a los 15, 30, 90, 180 y 270 días de la visita de inclusión.

En cada visita se realizó la valoración del estado de abstinencia/consumo del paciente. Para ello se partió del criterio de Volpicelli et $a^{14}$, que fijó como criterio de "Recaída" el consumo de > 5 consumiciones por ocasión de consumo ó > 5 días de ingestión de alcohol a la semana. Análogamente, como criterio de "Reanudación del consumo", se consideró la ingestión de 1 a 5 consumiciones de alcohol por ocasión de consumo o entre 1 y 5 días bebiendo a la semana.

De acuerdo con estos criterios relativos a la evolución de su consumo como variables principales, los pacientes se clasificaban en 3 grupos:

- Pacientes abstinentes: aquellos pacientes que no consumieron nada de alcohol a lo largo de todo el estudio.

- Pacientes que recaen: todos aquellos pacientes que realizaron consumo de $>05$ consumiciones por ocasión de consumo ó >5 días bebiendo a la semana, en algún momento del estudio.

- Pacientes que consumen: aquellos pacientes que refirieron consumo de 1 a 5 consumiciones de alcohol por ocasión de consumo o entre 1 y 5 días bebiendo a la semana, sin llegar a criterios de recaída en ninguna de las visitas.

Para el estudio del perfil del paciente respondedor a la naltrexona, se reagrupó a los pacientes, a partir de la $3^{a}$ semana de tratamiento (visita 3 , a los 30 días de la inclusión), en dos categorías superiores:

- pacientes respondedores: aquellos que se mantuvieron abstinentes o que reanudaron el consumo sin cumplir criterios de recaída $(n=160)$, y

- pacientes no respondedores: aquellos que cumplieron criterios de recaída en alguna de las visitas $(n=83)$.

Pacientes que abandonan o pérdidas: Aquellos que dejaron el seguimiento antes de la tercera visita y los pacientes que, aunque siguieron el estudio, dejaron de tomar naltrexona por su cuenta.

Otros parámetros evaluados en cada una de las visitas de seguimiento fueron:

Analítica completa.
Craving de los pacientes, evaluado mediante la Escala de Valoración del Deseo de Beber de Rubio ${ }^{22}$, que contiene los siguientes puntos de valoración: deseo de beber, deseo mientras bebe, deseo tras beber, sensación desagradable tras beber e intoxicación tras beber.

Estado de ansiedad. En cada una de las visitas de control, a excepción de la segunda (realizada a los 15 días), se valoraron mediante la Escala de Hamilton los síntomas de ansiedad presentes, tanto desde el punto de vista psicológico como somático.

Cumplimiento de las pautas terapéuticas prescritas.

Seguridad del tratamiento.

Valoración clínica global.

Los valores obtenidos se analizaron estadísticamente a partir de:

- Análisis del Perfil del Paciente: análisis descriptivo de las características basales de la muestra.

- Análisis de las variables de Seguimiento del Paciente: pruebas estadísticas Chi-cuadrado y no paramétrica de Wilcoxon en función del tipo de variable a analizar.

- Impresión Global: estadística descriptiva.

- Análisis del Perfil del Paciente Respondedor/no Respondedor: pruebas estadísticas Chi-cuadrado y t de Student, y las pruebas no paramétricas $U$ de Mann-Whitney y $\mathrm{H}$ de Kruskal-Wallis.

- Análisis de Seguridad: estadística descriptiva con la totalidad de los pacientes incluidos en el estudio.

Para el análisis estadístico de los resultados obtenidos tras el tratamiento con naltrexona, se utilizó una muestra de 304 pacientes, siendo excluídos 17 casos debido a:

a) falta de datos (8 casos), b) aparición de criterios de exclusión (1 caso por abandono del seguimiento durante dos visitas consecutivas y 3 casos por no tomar el tratamiento durante 15 días consecutivos), c) abandono voluntario del tratamiento (4 casos que, aunque siguieron en el estudio, dejaron el tratamiento farmacológico y cuyo número se estimó insuficiente para realizar ningún tipo de estadística comparativa sobre efectividad del tratamiento) y d) coincidencia de dos motivos (incumplimiento del tratamiento más de 15 días y abandono voluntario del tratamiento farmacológico), en un caso.

\section{RESULTADOS}

Se ha estudiado la evolución de estos pacientes desde la visita 3 hasta la visita 6 (a los 270 días de tratamiento); por tanto quedan excluidos 61 casos que 
abandonaron el estudio antes de la visita 3 (tabla 1), que marcaba el inicio del control de recaída.

\begin{tabular}{|c|c|c|}
\hline $\begin{array}{l}\text { Evolucö́n del consumo a lo lagodel } \\
\text { Estudio }\end{array}$ & n & $\%$ \\
\hline $\begin{array}{l}\text { no haconsumico } \\
\text { (abstinencia) }\end{array}$ & 138 & 45,4 \\
\hline ha consurido & 22 & 7,2 \\
\hline 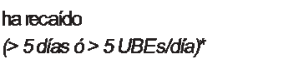 & 83 & 27,3 \\
\hline 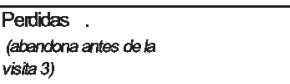 & 61 & 20.1 \\
\hline
\end{tabular}

Se ha constatado de nuevo la utilidad de la adición de naltrexona a las técnicas habituales de tratamiento del enfermo con dependencia alcohólica. Al finalizar el estudio, los pacientes de la muestra podían agruparse en tres categorías con arreglo a su consumo de alcohol durante los 9 meses de seguimiento:

- Abstinencia total o ningún consumo de alcohol en el $45,4 \%$ de los pacientes (138/304)

- Algún consumo en el 7,2\% de los pacientes (22/304)

- Recaída en el 27,3\% de los pacientes (83/304).

A su vez, cabe reagrupar a los pacientes en: "no respondedores" (27,3\%) y "respondedores" (52,6\%), según hubieran cumplido o no criterios de recaída. (La suma de porcentajes no totaliza cien, al haber excluido del análisis a los 61 pacientes que abandonaron antes de la $3^{a}$ visita, en que empezaba a regir el criterio de recaída.)

Ambos grupos de pacientes (respondedor / no respondedor) presentan valores similares en la mayoría de las variables sociodemográficas que describen la muestra (sexo, edad, nivel ocupacional, nivel socioeconómico, hábitat, estado civil, convivencia e iniciativa de la consulta) y únicamente presentan diferencias significativas en lo que hace referencia al nivel de estudios ( $p=0.039)$ y situación laboral $(p=0.041)$ (ver Tabla 2).

En el grupo de enfermos que no recaen hay mayor proporción de pacientes con nivel de estudios elementales que en el grupo de los que sí recaen $161 \%$ vs 49,4\%), mientras que existe una menor proporción de pacientes con estudios de bachillerato y superiores en los que no recaen que en el los que sí recaen (28.9\% vs $33.3 \%$ y $7.5 \%$ vs $17.3 \%$ respectivamente).

Respecto a la situación laboral, observamos que en el grupo de los que no recaen hay una mayor proporción de pacientes en activo y parados que en los que sí recaen $(65 \%$ vs $57.8 \%$ y $20.6 \%$ vs $16.9 \%$, respectivamente) y una menor proporción de pensionis- tas y amas de casa que en los que sí recaen $(9.4 \%$ vs $12 \%$ y $5 \%$ vs $8.4 \%$, respectivamente).

No se observan diferencias entre los dos grupos en lo que hace referencia a los criterios diagnósticos, gravedad de la dependencia alcohólica y la dependencia fisiológica.

Ninguno de los dos grupos presenta una mayor proporción de antecedentes médicos, psiquiátricos o familiares, ni tampoco respecto a las patologías actuales (médicas o psiquiátricas).

No se observan diferencias entre el grupo que recae y el que no recae en lo referente al consumo de alcohol ni de otras sustancias. Tampoco se observan diferencias significativas respecto al tratamiento del alcoholismo (desintoxicación, intervención psicosocial) y otros tratamientos concomitantes ni en la Escala de Valoración del Deseo de Beber de Rubio.

La escala de valoración de la ansiedad de Hamilton muestra diferencias entre los pacientes que recaen y que no recaen que, aunque estadísticamente alcanzan la significación estadística, clínicamente son irrelevantes (media del número de síntomas: 8,49 \pm 3,72 vs $9,69 \pm 3,83$ y media del número de síntomas psicológicos 4,88 $\pm 2,01$ vs 5,55 $\pm 1,65)$.

Comparación del perfil basal de los pacientes que finalizan el estudio versus abandonos/pérdidas:

Respecto al grupo de 61 pacientes que abandonan el estudio antes de realizar la visita 3 (a los 30 días de la inclusión) sólo se observan diferencias significativas, con respecto al grupo de pacientes que continúan en tratamiento, en lo que hace referencia al nivel socioeconómico y al hábitat.

En el grupo que abandonan el estudio antes de la visita 3 (primer mes de tratamiento) existe un mayor porcentaje de pacientes con nivel socioeconómico bajo que en el grupo de los que continúan (48,3\% vs $31,1 \%)$, mientras que, en el grupo de pacientes que continúan el estudio más allá de la visita 3 , existe una mayor porcentaje de pacientes con nivel socioeconómico medio (61,3\% vs 51,7\%).

Respecto al hábitat, cabe destacar que el 47,5\% de los pacientes que abandonan viven en ciudades de entre 10.000 y 100.000 habitantes y un $24,6 \%$ en ciudades entre 100.000 y 1.000 .000 de habitantes, mientras que de los pacientes que siguieron en el estudio sólo un 26,7\% viven en ciudades entre 10.000 y 100.000 habitantes y un $32,9 \%$ viven en ciudades entre 100.000 y 1.000 .000 de habitantes.

\section{Resultados del Tratamiento}

Para la estadística descriptiva y comparativa del resultado del tratamiento se han establecido tres grupos de pacientes: pacientes abstinentes, pacientes que consumen (cumplen criterios de reanudación del 
Tabla 2. Características basales del grupo de pacientes que recaen v/s pacientes que no recaen: Datos sociodemográficos

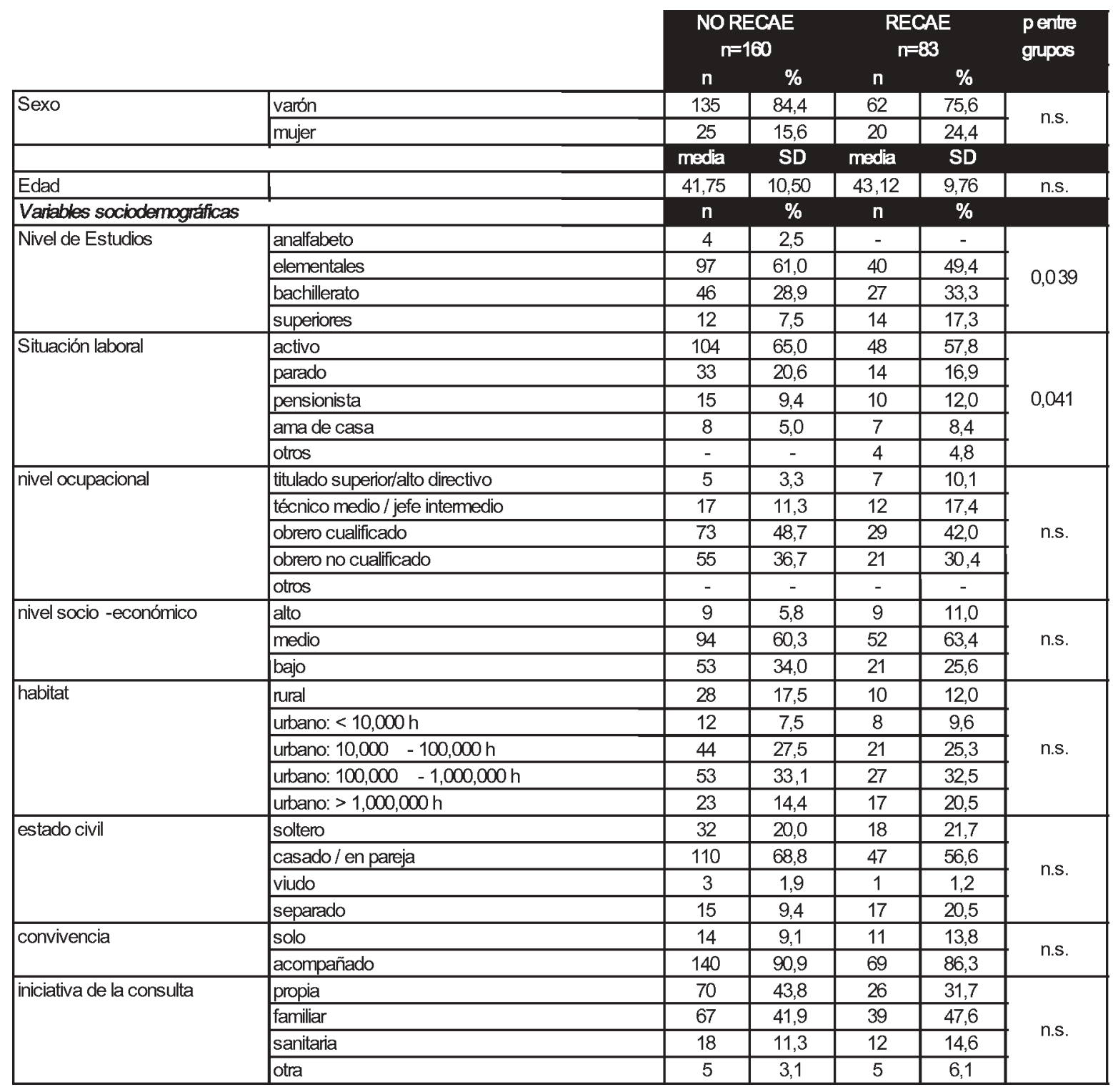

consumo pero no de recaída) y pacientes que recaen (cumplen criterios de recaída).

Con respecto a la evolución del consumo, en la tabla siguiente (tabla 3) se puede observar, sobre el total de pacientes que siguen en el estudio en cada visita, la proporción de pacientes abstinentes, de pacientes que reanudan el consumo de alcohol o de pacientes que cumplen criterios de recaída visita a visita. Dado que el criterio de recaída se establece a partir de la $3^{\mathrm{a}}$ semana de tratamiento, se ha estudiado la evolución de los pacientes desde la visita 3 (a los 30 días de tratamiento) hasta la visita 6 (a los 270 días de tratamiento):

\section{Tabla 3: Evolución del consumo. Recaídas y consumos, visita a visita}

\begin{tabular}{|l|cc|cc|cc|cc|}
\hline & \multicolumn{2}{|c|}{$\begin{array}{c}\text { VISITA 3 } \\
\mathbf{n = 2 4 3}\end{array}$} & \multicolumn{2}{|c|}{$\begin{array}{c}\text { VISITA 4 } \\
\mathbf{n = 1 9 4}\end{array}$} & \multicolumn{2}{c|}{$\begin{array}{c}\text { VISITA 5 } \\
\mathbf{n = 1 5 1}\end{array}$} & \multicolumn{2}{c|}{$\begin{array}{c}\text { VISITA 6 } \\
\mathbf{n = 1 0 3}\end{array}$} \\
\hline & $\boldsymbol{N}$ & $\mathbf{\%}$ & $\boldsymbol{n}$ & $\mathbf{\%}$ & $\boldsymbol{n}$ & $\mathbf{\%}$ & $\boldsymbol{n}$ & $\mathbf{\%}$ \\
\hline Abstinentes & 195 & 80,2 & 132 & 68,0 & 100 & 66,2 & 79 & 76,7 \\
Consumo sin criterios de recaída & 15 & 6,2 & 17 & 8,8 & 7 & 4,6 & 10 & 9,7 \\
Recaen (> 5 días ó > 5 UBEs/día) & 33 & 13,6 & 45 & 23,2 & 44 & 29,1 & 14 & 13,6 \\
\hline
\end{tabular}


La tasa de cumplimentación del tratamiento con naltrexona (toma de la medicación superior al 80\%) a lo largo del estudio (visita 3 vs. visita 6) se situó, para el grupo de los abstinentes, entre el $82,7 \%$ y el 86,9\%; para el grupo de los que han realizado algún consumo, entre el $72,7 \%$ y el $96 \%$, y, para el grupo de los que recaen, entre el el 52,6\% y el 82,3\%.

La cantidad media de alcohol (UBE) consumida, en las visitas 4, 5 y 6 es superior en el grupo de pacien- tes que recaen que en los que consumen sin recaer ( $p=0,003, p=0,001$ y $p=0,017$ respectivamente). En el grupo de pacientes que recaen se observa que el incremento en la cantidad de alcohol (UBE) consumida de visita a visita alcanza la significación estadística entre las visitas 3 y $4(p=0,000)$, es decir, al primer y tercer mes de tratamiento, no observándose esta significación en el resto de visitas ni en el grupo de los pacientes que consumen. (Figura 1)

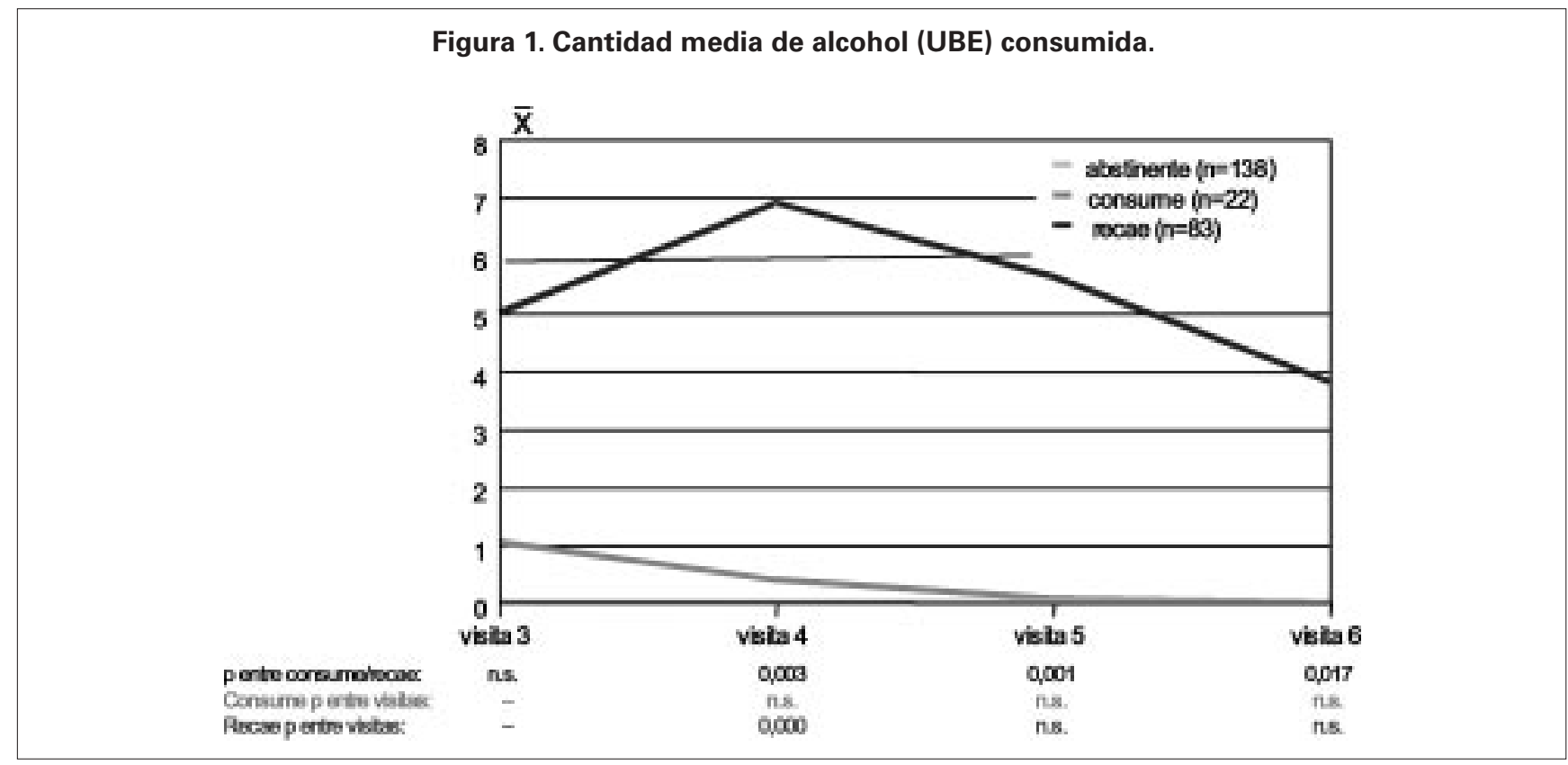

La media de los días de consumo a la semana en las visitas 4, 5 y 6 es superior en el grupo de pacientes que recaen que en los que consumen $(p=0,020$; $p=0,002 ; p=0,013$, respectivamente). Sin embargo, la media de días de consumo semanales tiende a disminuir a lo largo del estudio, llegando a cero en la visita
5 para el grupo que había realizado algún consumo en los periodos de valoración anteriores a los 180 días. Esta disminución es estadísticamente significativa entre las visitas 3 y $4(p=0,000)$ y entre las visitas 5 y $6(p=0,05)$ en el grupo de los pacientes que recaen. (Figura 2).

Figura 2. Media de los días de consumo de alcohol a la semana.

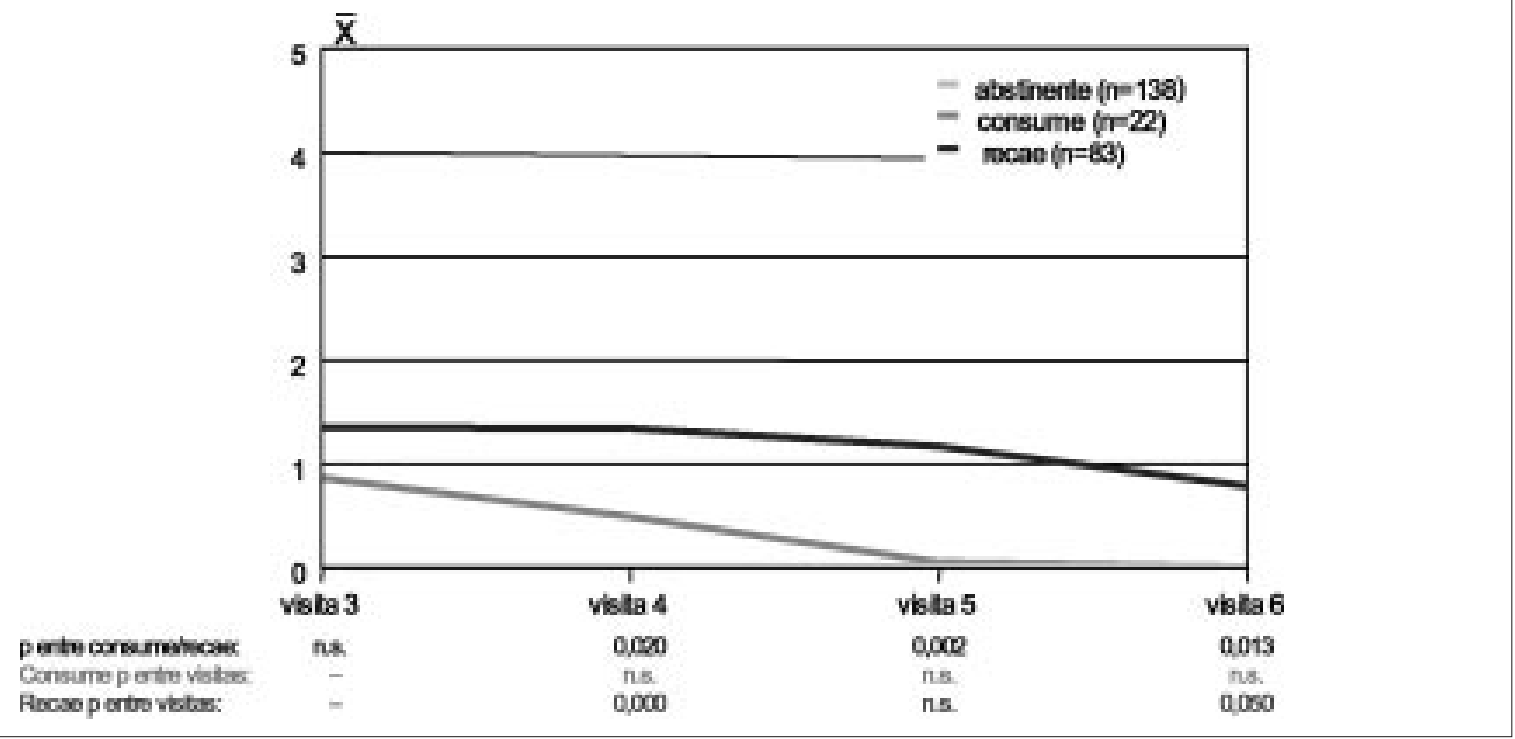


Por lo que respecta a los episodios de consumo de alcohol, (Figura 3), en la visita 5, a los 180 días de tratamiento, la media de episodios de consumo es superior en el grupo de pacientes que recaen que en los pacientes que consumen. No obstante, y al igual que ocurre con los días de consumo, la media de episodios de con- sumo de alcohol, tanto en el grupo que recae como en el grupo que realiza algún consumo, tiende a disminuir a lo largo del estudio, llegando a abandonar todo el consumo entre las visitas 5 y 6, quienes habían consumido sin cumplir criterios de recaída, aunque la diferencia no alcance significación estadística.

Figura 3. Media del número de episodios de consumo de alcohol.

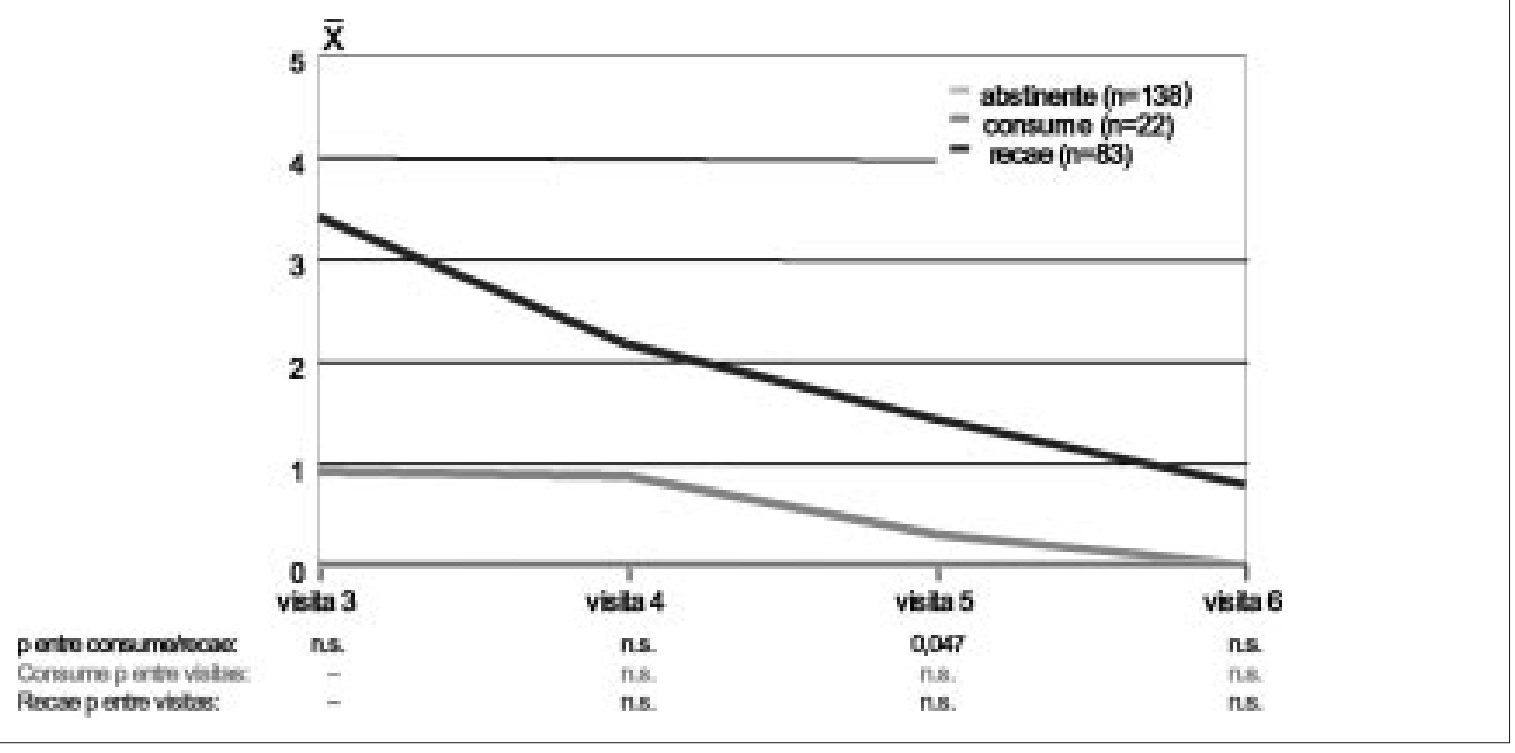

En contra de lo que se esperaba, el porcentaje de pacientes que bebieron y recayeron fue superior al de aquéllos que reanudaron el consumo sin llegar a cumplir criterios de recaída en cada visita. Sin embargo, el porcentaje de estos últimos tiende a aumentar mientras el de los pacientes recaídos tiende a disminuir, siendo en el mes $9^{\circ}$ ( $6^{a}$ visita) cuando más favorable es el balance entre quienes consumen y quienes recaen.

Por lo que se refiere al deseo de beber, disminuye a medida que avanza el estudio en los pacientes que permanecen abstinentes (ítem 1 de la Escala de Rubio).

En el grupo de pacientes que recaen, se observa que, en la visita 2, se produce un incremento significativo del número de pacientes que no tienen deseo de beber, un aumento de la proporción de pacientes con un deseo de beber leve/moderado y una disminución de la proporción de pacientes que tienen un deseo intenso/muy intenso/ el más intenso de beber; manteniéndose estas proporciones a lo largo del estudio.

La escala de ansiedad de Hamilton mostró una disminución significativa del número total de síntomas en los tres grupos de pacientes a lo largo del estudio $(p<0,05)$. Comparando los tres grupos, vemos que el grupo de sujetos que recaen muestran más síntomas en la visita inicial $(p=0,020)$, principalmente a expensas de los síntomas psicológicos $(p=0,017)$. En la visita final, el grupo de pacientes abstinentes presenta un menor número de síntomas totales, psicológicos y somáticos que el resto de grupos ( $p=0,000$ en los tres tipos de síntomas). La puntuación media de la escala de ansiedad de Hamilton muestra una disminución significativa de la intensidad de la ansiedad para cada grupo de pacientes, comparando la visita inicial y la final ( $p=0,000$ para los abstinentes y los que recaen $y$ $\mathrm{p}=0,012$ para los que consumen).

La impresión global sobre la evolución de la enfermedad, expresada por el médico y el paciente, es de una mejoría subjetiva de la patología, en casi el $80 \%$ de casos, a lo largo de todas las visitas excepto en la cuarta, cuando la proporción de vañloraciones positivas disminuyó sin llegar a alcanzar significación estadística.

No se ha podido cumplir el objetivo de comparar el beneficio de la naltrexona asociada a las técnicas habituales de tratamiento frente a estas técnicas solas en aquellos pacientes que abandonaron voluntariamente la naltrexona, ya que sólo hubo 4 casos en los que constara el abandono del fármaco y que siguieran asistiendo a las visitas de seguimiento durante el tiempo en que permaneció abierto el estudio.

En relación a la Seguridad del tratamiento farmacológico durante el estudio, solo en un total de 15 pacientes $(4,7 \%)$ se refirieron reacciones adversas, ninguna de ellas grave. En la mayoría de casos (10 pacientes), las reacciones adversas se presentaron en la visita 2, a los 15 días de iniciar el tratamiento.

Las reacciones adversas recogidas en la visita 2 fueron: taquicardia (el primer día de tratamiento 
cediendo a las 24 horas); estado confusional, ataxia y temblor leves (al asociar naltrexona con acamprosato, remitiendo espontáneamente a las 24 horas); disminución de reflejos, enlentecimiento psicomotor y sensación de extrañeza (aparición a los 15 días del tratamiento y recuperándose después); diarrea e intranquilidad (remitieron al suspender la medicación); insomnio, debilidad, anorexia, dispepsia importante, alteraciones bucales, cansancio y ligeros mareos. En la visita 3, sólo 3 pacientes refirieron presentar reacciones adversas consistentes en ligeros mareos. En la visita 5 (tras 180 días de tratamiento), dos pacientes presentaron reacciones adversas, uno en forma de anorexia y fatigabilidad y el otro con aumento de las cifras de GGT en la analítica.

Sólo se describieron las reacciones adversas de 11 de los 15 pacientes. Tres casos se recuperaron, 3 persistieron y en 5 casos se desconoce la evolución.

\section{DISCUSIÓN}

Con los datos de que se dispone, no es posible describir el perfil de paciente con dependencia de alcohol que se beneficiaría más del uso de naltrexona en la fase de deshabituación.

La dificultad de definir un perfil de paciente que se beneficiaría más de la naltrexona ya había sido señalada por Arias et al. ${ }^{22}$. En su estudio GEODA, los autores señalaban la heterogeneidad de la muestra, de los tratamientos coadyuvantes y de los investigadores, así como la falta de grupo control, como importante limitación a la hora de identificar factores predictores de resultado. Asimismo, subrayaban la imposibilidad de valorar si dichos factores se refieren al resultado del tratamiento con naltrexona o al del conjunto de medidas terapéuticas. En cualquier caso, el estudio GEODA $^{22}$ apuntaba la menor gravedad de la dependencia, el uso de medidas psicoterapéuticas y el consumo no diario de alcohol como predictores de respuesta favorable. El presente estudio no ha corroborado ninguna de estos factores, aunque apunta en la misma dirección. Tampoco hemos comprobado que la respuesta terapéutica sea superior con la administración conjunta de naltrexona y un aversivo ${ }^{23}$, además de la naltrexona

El estudio de Jiménez Arriero et al. ${ }^{8}$, cuyo objetivo era describir el perfil de los pacientes que se hallaban por primera vez en tratamiento con naltrexona, revela que éstos solían ser varones más jóvenes (en la cuarentena), con mejores circunstancias socio-sanitarias y mayor pérdida de control que la media de pacientes en tratamiento aportada por la bibliografía y los datos de la población española. La diferencia fundamental entre dicho estudio y el nuestro, es que el de Jiménez Arriero et al. ${ }^{8}$ nos muestran la tendencia a prescribir naltrexona a un determinado perfil de paciente, en tanto que nuestro estudio y el de Arias et al. ${ }^{22}$ intentaban comprobar si existe realmente un perfil de pacientes que se beneficien en mayor medida de la naltrexona.

El interés de los estudios prospectivos, naturalistas y no controlados radica en que sus resultados son más extrapolables a las condiciones de la práctica clínica real, aunque introducen limitaciones a la hora de atribuir resultados.

El presente trabajo se diseñó como un estudio fase IV y no se proponía, por tanto, estudiar la eficacia del tratamiento con naltrexona, reiteradamente demostrada en ensayos clínicos previos ${ }^{12-20,23,25}$. Sin embargo se había previsto comprobar adicionalmente la utilidad del tratamiento con naltrexona en el tratamiento de los pacientes alcohólicos, a ser posible analizando también su efectividad frente al grupo de pacientes que abandonaron la naltrexona pero siguieron en programa de deshabituación (grupo control). Este aspecto no ha podido ser cumplimentado por ausencia de un número suficiente de sujetos control.

A pesar de esta indiscutible limitación, los resultados obtenidos revelan la utilidad del tratamiento y la contribución de naltrexona a la efectividad del tratamiento habitual del alcoholismo, reafirmando los datos que aporta la literatura nacional e internacional.

Un aspecto novedoso de este estudio es su larga duración, muy superior a los 3 meses de la inmensa mayoría de estudios internacionales y la más larga en nuestro país, que indica el superior beneficio de tratamientos más prolongados. Por otra parte, la muestra de pacientes que ha participado en este estudio es la mayor de cuantas se hayan analizado en los estudios con naltrexona publicados hasta la fecha.

También es importante destacar la circunstancia de ser este un estudio multicéntrico realizado en nueve comunidades autónomas de todo el estado español contemplando, en la medida de lo posible, las diferenciales socioculturales tanto en la cantidad como en el modo de los españoles ya señaladas en anteriores estudios sobre hábitos de consumo ${ }^{27}$.

A los 9 meses de tratamiento, el 52,6\% de pacientes aparece como "respondedor"al tratamiento, ya sea con abstinencia total $(45,4 \%)$ o con algún consumo sin recaer $(7,2 \%)$.

A lo largo del tiempo, tienden a disminuir tanto los consumos de alcohol, como el número de días de consumo por semana. La mayoría de las recaídas se producen entre los 3 y los 6 meses de tratamiento. Transcurridos 9 meses de tratamiento con naltrexona, se observa un aumento en la proporción de pacientes que consumen alcohol no llegando a criterios de recaída.

El tratamiento con naltrexona presenta una excelente tasa de cumplimiento $(79,9 \%)$, superior a otros estudios españoles como el del grupo GEODA ${ }^{22}$, y también internacionales ${ }^{24}$. Los abandonos de trata- 
miento fueron bajos $(20,1 \%)$, bastante inferiores a los observados en la práctica clínica habitual en los tratamientos estándar con pacientes alcohólicos y también a los señalados en publicaciones anteriores sobre efectividad de la naltrexona ${ }^{25}$.

La naltrexona es un fármaco con una elevada seguridad, observándose únicamente un 4,7\% de casos de reacciones adversas, ninguna de las cuales se consideró relevante desde el punto de vista clínico. Se corrobora de nuevo el elevado margen de seguridad en la administración prolongada de naltrexona, ya señalado por Ochoa et al. ${ }^{26}$ a los 6 meses de tratamiento.

Los resultados obtenidos nos llevan, pues, a concluir que, si bien no se ha podido describir el perfil del paciente candidato ideal para el tratamiento con naltrexona, la prescripción de dicho fármaco aporta unos beneficios que pueden maximizarse prolongando la farmacoterapia a lo largo de un año, lo que coincide con la parte intensiva de cualquier tratamiento de deshabituación y es coherente con la concepción del proceso de cambio.

\section{BIBLIOGRAFÍA}

(1) Miller, NS, Millman RB: A common cause of alcoholism. J of Substance Abuse Treatment, 41-43, 1989.

(2) Erickson CK. Review of neurotransmitters and their role in alcoholism treatment. Alcohol and Alcoholism 1996; 31 (suppl 1): 5-11.

(3) Chick J, Erickson CK. Conference summary: consensus conference on alcohol dependence and the role of pharmacotherapy in its treatment. Alcoholism: Clinical and Experimental Research 1996; 20: 391-402.

(4) Torres Hernández MA. Evolución tras 2 años de tratamiento de un grupo de alcohólicos. Psiquiatría Biológica 1998; 5 (supl 1): 54-60.

(5) Anton RF, Kranzler HR, Meyer RE. Neurobehavioral aspects of the pharmacotherapy of alcohol dependence. Clin Neuroscience 1995; 3: 145-154.

(6) Volpicelli JR, Ulm RR, Hopson N. Alcohol drinking in rats during and following morphine injections. Alcohol, 1991; 8:289-292.

(7) Reid LD, Hunter GA. Morphine and naloxone modulate intake of ethanol. Alcohol, 1984; 1: 33-37.

(8) Jiménez-Arriero, M.A; Guardia, J; Bravo de García-Quijada, JL. Características clínicas y utilización de Naltrexona en la deshabituación alcohólica: Estudio stop. Adicciones 2000; Vol.12.

(9) Samson HH, Doyle TF. Oral ethanol self-administration in the rat: Effect of naloxone. Pharmacol Biochem Behav, 1985; 22: 91-99.

(10) Volpicelli JR, Davis MA, Olgin JE. Naltrexone blocks the post-shock increase of ethanol consumption. Life Sci, 1986; 38: 841-847.
(11) Reid LD. Endogenous opioids and alcohol dependence: opioid alkaloids and the propensity to drink alcoholic beverages. Alcohol, 1996; 13: 5-11.

(12) Terenius L. Alcohol addiction (Alcoholism) and the opioid system. Alcohol, 1996; 13: 31-34

(13) Volpicelli JR, Alterman Al, Hayashida M, o’Brien ChP. Naltrexone in the treatment of alcohol dependence. Arch Gen Psychiatry 1992; 49: 876-880.

(14) O'Malley SS, Jaffe AJ, Chang G et al. Naltrexone and coping skills therapy for alcohol dependence: A controlled study. Arch Gen Psychiatry 1992; 49: 881-887.

(15) Volpicelli RF, Watwon NT, King AC et al. Effect of naltrexone on alcohol "high" in alcoholism. Am J Psychiatry 1995; 152: 613-615.

(16) Volpicelli RF, Karen LC, Watson NT, o'Brian ChP. . Effect of naltrexone in the treatment of alcoholism: Predicting response to naltrexone. J Clin Psychiatry 1995b; 56 (suppl 7): 39-44

(17) O’Brian CP, Volpicelli LA, Volpicelli JR. Naltrexone in the treatment of alcoholism : A Clinical Review Alcohol 1996; 13: 35-49.

(18) O’Malley SS, Jaffe AJ, Rode S, Rounsaville BM. Experience of a "Slip" among alcoholics treated with naltrexone or placebo. Am J Psychiatry 1996; 153: 281-283.

(19) O’Malley SS, Jaffe AJ, Chang G, Rode S et al. Six-month follow-up of naltrexone and psychotherapy for alcohol dependence. Arch Ge Psychiatry 1996b; 53: 217-224.

(20) Davidson D, Swift R, Fitz E. Naltrexone increases the latency to drink alcohol in social drinkers. Alcohol Clin Exp Res 1996; 20: 732-739.

(21) Rubio Valladolid, G; López Ruiz, M.:"Validación del cuestionario sobre los componentes obsesivo-compulsivo de bebida en alcohólicos españoles". Adicciones 1999;. 11 (1):7-15.

(22) Arias F, Ochoa E, Torres MA and GEODA Group: Predictors of response to treatment with naltrexone in alcoholism. Trastornos Adictivos 2000; 2 (3): 194-200.

(23) Landabaso MA, Iraurgi I, Sanz J, Fernández de Corres B, Ruíz de Apodaka J, Jiménez-Lerma JM et al.: Naltrexona más aversivo en alcohólicos refractarios a tratamiento. Psiquiat Biol 1997; 4: 5-8.

(24) Croop, R., Fralkner,e., Labriola,D. The safety profile of naltrexone in the treatment of alcoholism. Results from a multicenter usage estudy. Arch. Gen. Psychiatry 1997; 54; 1130-1135.

(25) Torres, M.A. ;Arias, F.; Ochoa, E.: Efectividad de la naltrexona en pacientes alcohólicos. Adicciones. 2000.Vol 12. $n^{\circ} 2$. pp 49-57.

(26) Ochoa E, Arias F, Torres MA.: Evaluación de la seguridad del tratamiento con naltrexona en la dependencia del alcohol. Actas Esp Psiquiatr 2000; 28 (3): 161-168.

(27) Llopis,J.J., Rodriguez Martos, A., Gual, A.: Registro del consumo de bebidas alcohólicas mediante la unidad de bebida estándar. Diferencias geográficas. Adicciones. Vol 12, n 1. pp 11-21. 
